\title{
analytical chemistry
}

Subscriber access provided by Library, Special Collections and Museums, University of Aberdeen

\section{Article}

\section{AF4-UV-MALS-ICP-MS/MS, spICP-MS and STEM-EDX for the characterization of metal-containing nanoparticles in gas condensates from petroleum hydrocarbon samples}

Daniel Ruhland, Kenneth Chinedu Nwoko, Magali Perez, Joerg Feldmann, and Eva M. Krupp

Anal. Chem., Just Accepted Manuscript • DOI: 10.1021/acs.analchem.8b05010 • Publication Date (Web): 05 Dec 2018

Downloaded from http://pubs.acs.org on December 9, 2018

\section{Just Accepted}

"Just Accepted" manuscripts have been peer-reviewed and accepted for publication. They are posted online prior to technical editing, formatting for publication and author proofing. The American Chemical Society provides "Just Accepted" as a service to the research community to expedite the dissemination of scientific material as soon as possible after acceptance. "Just Accepted" manuscripts appear in full in PDF format accompanied by an HTML abstract. "Just Accepted" manuscripts have been fully peer reviewed, but should not be considered the official version of record. They are citable by the Digital Object Identifier (DOI囚). "Just Accepted" is an optional service offered to authors. Therefore, the "Just Accepted" Web site may not include all articles that will be published in the journal. After a manuscript is technically edited and formatted, it will be removed from the "Just Accepted" Web site and published as an ASAP article. Note that technical editing may introduce minor changes to the manuscript text and/or graphics which could affect content, and all legal disclaimers and ethical guidelines that apply to the journal pertain. ACS cannot be held responsible for errors or consequences arising from the use of information contained in these "Just Accepted" manuscripts. 


\title{
AF4-UV-MALS-ICP-MS/MS, spICP-MS and STEM-EDX for the characterization of metal-containing nanoparticles in gas condensates from petroleum hydrocarbon samples
}

\author{
Daniel Ruhland*, Kenneth Nwoko, Magali Perez, Jörg Feldmann, and Eva M. Krupp \\ Trace Element Speciation Laboratory (TESLA), University of Aberdeen, Aberdeen AB24 3UE, UK
}

\begin{abstract}
The coupling of flow field flow fractionation (FlFFF) with ICP-MS/MS for the fractionation and analysis of natural nanoparticles in environmental samples is becoming more popular. However, the applicability of this technique to non-aqueous samples such as gas condensates from petroleum hydrocarbon samples has not been reported yet. In this study, an asymmetric flow-field flow fractionation (AF4) system coupled with UV and MALS detectors has been optimized to perform the fractionation of natural nanoparticles present in a gas condensate sample, using THF as the carrier liquid. Prior to this, STEM images indicated the presence of both large (200 nm and more) and smaller (50 nm and less) particles, whose irregular shape is probably due to agglomeration. AF4-UV-MALS-ICP-MS/MS confirmed the presence of various nanoparticles and colloids, some containing aromatic compounds as well as various metals including $\mathrm{Hg}$. The recovery against an injection without crossflow is around $75 \%$ for most metals. The presence of $\mathrm{Hg}$ containing nanoparticles was confirmed with offline single particle ICP-MS (spICP-MS), using THF as a solvent. These NPs were identified as HgS using STEM-EDX. These results highlight for the first time that particulate matter may contaminate gas condensates with a series of elements (Al, P, S, Ti, V, Mn, Fe, Co, Cu, Zn, As, Se, Cd, Hg, Pb) which can make the upstream use problematic, especially for mercury.
\end{abstract}

\section{INTRODUCTION}

As conventional petroleum sources are increasingly difficult to exploit, gas condensates are becoming viable options for the petroleum industry. From $5.8 \mathrm{Mb} / \mathrm{d}$ in 2006 , the global condensate production is expected to reach $9.1 \mathrm{Mb} / \mathrm{d}$ in 2020 according to an International Energy Agency (IEA) projection ${ }^{1}$. A gas condensate mostly contains C5+ hydrocarbons and shares many of the characteristics of a light crude oil. It generally occurs in association with natural gas and condenses as the gas rises to the surface. Petroleum liquids often contain minor amounts of impurities; in 
particular, mercury is present in almost all hydrocarbon sources worldwide, in concentration ranging from 10 to 3,000 $\mu \mathrm{g} \cdot \mathrm{kg}^{-1}$ depending on geological location ${ }^{2}$. The origin of mercury is believed to be a combination of atmospheric mercury deposition and secondary geological processes ${ }^{3}$.

Mercury-containing hydrocarbons have the potential to cause corrosion of welds, cryogenic components, and aluminum based heat exchangers ${ }^{4}$. Mercury can also affect the efficiency of platinum group metals catalysts, as even $\mu \mathrm{g} . \mathrm{kg}^{-1}$ levels can lead to catalyst poisoning ${ }^{5}$. Last but not least, environmental issues due to workers exposure and release to the environment are also present. These concerns have led some refiners to impose limits for total mercury on the feedstock in the single $\mu \mathrm{g} \cdot \mathrm{kg}^{-1}$ range $^{6}$. For fields with a high mercury concentration it may be necessary to add a mercury removal system into the process design. Such systems usually rely on surface adsorption on metal sulfide pellets, e.g. sulfide-containing alumina ${ }^{7}$. However, individual $\mathrm{Hg}$ species exhibit different chemical and physical properties which may affect their reactivity towards the adsorbent. It has indeed been observed that current mercury removal systems typically remove elemental mercury only. For this reason, there is a need to identify exactly which species of mercury are present. The total amount of $\mathrm{Hg}$ should fulfil the following mass balance equation ${ }^{8}$ :

$$
H g_{\text {total }}=\mathrm{Hg}^{0}+\mathrm{R}_{2} \mathrm{Hg}+\mathrm{RHg} \mathrm{g}^{+}+\mathrm{Hg}^{2+}+H g_{\text {particulate }}
$$

To the best of our knowledge, mercury speciation studies in gas condensates have mainly been focused on elemental, organic and inorganic mercury, mainly relying on separation by Gas or Liquid Chromatography and detection by ICP$\mathrm{MS}^{9}$. The particulate $\mathrm{Hg}$ is then calculated as the difference between total $\mathrm{Hg}$ and the sum of all the other species. More specifically, no information is found in the literature about the presence of naturally occurring $\mathrm{Hg}$ nanoparticles (NPs) in condensates. While determining the concentration of $\mathrm{Hg}^{2+}$ species in a crude oil sample using species-specific isotope dilution (SS-ID-MS) Gajdosechova et al. ${ }^{8}$ reported random variations which were attributed to the presence of $\mathrm{HgS}$ nanoparticles in the sample; Recently, Avellan et al. highlighted the presence of HgS NPs in solid petroleum residues ${ }^{10}$. Therefore, the presence of Hg-containing NPs in gas condensates seems plausible as well.

The increasing importance of engineered NPs and concerns about their potential toxicity have led to the development of analytical methods in order to characterize and quantify nano-scale dispersed materials in environmental samples. Key challenges include potentially complex matrices, numerous measurands involved (e.g. size distribution, chemical composition, shape), and the dynamic nature of NPs solutions due to aggregation, dissolution or surface modification ${ }^{11}$. 
Several established NPs characterization techniques are currently availablel ${ }^{12,13}$. This includes microscopy techniques (electron microscopy and atomic force microscopy), and light scattering techniques. More recently, the sensitivity and elemental specificity of inductively coupled plasma-mass spectrometry (ICP-MS) has been adapted to the analysis of NPs. So-called single particle inductively coupled plasma-mass spectrometry (spICP-MS) rapidly provides information regarding size, size distribution, particle number concentration, and elemental composition, as well as possible aggregation processes ${ }^{14}$.

The coupling of Flow Field-Flow Fractionation (FlFFF), and particularly asymmetric FlFFF (AF4), with varying techniques such as UV/fluorescence ${ }^{15,16,14}$, multi-angle light scattering (MALS) ${ }^{17-19}$, and ICP-MS ${ }^{20-22}$, is also becoming increasingly popular. FlFFF is a chromatography-like particle separation technique based on the injection of a sample into a thin chamber through which a carrier liquid is flowing. The separation is achieved by a perpendicular liquid flow called cross flow ${ }^{23}$ : due to the diffusion phenomenon and parabolic flow profile inside the channel, small particles elute earlier than bigger ones. The "Achilles heel" of FlFFF is the sample-membrane interaction, which can cause significant sample losses due to permeation of NPs through the membrane and adsorption onto the membrane. A few studies using the AF4 with organic solvents for polymer separation are mentioned in the literature ${ }^{19,24}$; however this technique has, to the best of our knowledge, never been applied to petroleum matrices so far, and especially gas condensates and never been coupled to ICPMS for element-specific detection.

The aim of this study was to investigate the use of AF4 with on-line MALS, UV and ICP-MS for the characterization of natural nanoparticles in petroleum hydrocarbon samples. This technique has also been challenged against STEM and off-line spICP-MS measurements. Increased focus has been placed on $\mathrm{Hg}$ nanoparticles, whose presence in gas condensates is the most concerning.

\section{EXPERIMENTAL SECTION Reagents and samples.}

All chemicals used were of analytical reagent grade, unless stated otherwise. For AF4-ICP-MS, both o-xylene (Sigma Aldrich, UK) and THF (Perkin Elmer, UK) were used as carrier liquids. A polystyrene particle standard (PSS 62.5 kDa, PostNova Analytical, Germany) with a diameter of $23 \mathrm{~nm}$ was used to normalize the MALS detector. For spICP-MS, the samples were diluted in THF, which was also used as a blank solution. Gold nanospheres coated with polystyrene were purchased from nanoComposix. The gold NPs had a nominal diameter of $100 \mathrm{~nm}$ and were used for the determination of the nebulization efficiency after dilution to approximately $300 \mathrm{ng} . \mathrm{L}^{-1}$ (around $7.5 \times 10^{-7}$ particles/L) in 
THF. Dissolved elemental organic standards (Conostan S21+ Hg, S and Se standards, Canada) at a concentration of 5 $\mu \mathrm{g} . \mathrm{kg}^{-1}$ were prepared in THF or o-xylene from the $100 \mathrm{mg} \cdot \mathrm{kg}^{-1}$ standards in blank oil and used to measure elemental response factors. All dilutions were carried out gravimetrically. The samples were sonicated for 10 minutes before analysis.

The gas condensate sample (“Condensate B", geographical location not disclosed) presented in this study was provided by Johnson Matthey, UK, and stored in a $100 \mathrm{~mL}$ amber glass bottle in the dark at room temperature. Total concentrations for several elements were measured by ICP-MS using direct dilution in o-xylene, as described by Poirier et a ${ }^{25}$. The results are displayed in Table $\mathrm{S} 1$.

\section{Analytical methods.}

STEM analysis. The sample was sonicated for 10 minutes and a $5 \mu \mathrm{L}$ aliquot was fixed on a circular, Formvar-coated 200 mesh copper grid with a diameter of $3.05 \mathrm{~mm}$. Microscopy was performed at the Johnson Matthey Technology Centre in Sonning (U.K.). The images were obtained with a JEM 2800 (Scanning) Transmission Electron Microscope, at an accelerating voltage of $200 \mathrm{kV}$ and were analyzed using the software package JEOL analysis station. The particle size distribution was obtained using the open source image processing program ImageJ. Briefly, a FFT bandpass filter is applied to increase the contrast between the particles and the background. The software then delimits each particle and calculates the particle area, from which the particle diameter (or equivalent circular diameter, if the particle is not round) can be deduced. Compositional analysis has been performed by X-ray emission detection in the scanning mode.

spICP-MS analysis. The nanoparticles analysis were performed on a 7900 ICP-MS from Agilent Technologies (Tokyo, Japan). Analysis was performed in fast time resolved analysis with an integration time of $0.1 \mathrm{~ms}$. The main parameters are detailed in Table S2. Lenses parameters were optimized daily to reach the maximum sensitivity by using a tune solution prepared by dissolving Conostan $($ elemental standards in THF. The data analysis was performed using the optional single nanoparticle application module of the ICP-MS MassHunter software (Agilent Technologies).

AF4-ICP-MS/MS coupling. The FlFFF separation was carried with an AF 2000 asymmetric flow field flow fractionation (AF4) system (PostNova Analytics, Landsberg, Germany), combined with the AF2000 Control Program interface (v. 2.0.7.0). A temperature of $10^{\circ} \mathrm{C}$ was maintained in the autosampler for this experiment, while the channel was kept at $25^{\circ} \mathrm{C}$. Three pumps were used: two isocratic pumps to provide the tip flow and focus flow, while a Kloehn 
syringe pump provided the crossflow. The degasser was placed between the carrier liquid reservoir and the isocratic pumps. Two filters were placed in-line after each pump and changed regularly.

In $\mathrm{AF} 4$, separation is achieved in a flat channel by means of an externally generated cross flow applied in a perpendicular direction across a flat semi-permeable membrane. The AF4 was fitted with a trapezoid channel with a length of $275 \mathrm{~mm}$, and width of $20 \mathrm{~mm}$ and $5 \mathrm{~mm}$, with a ceramic frit and a $350 \mu \mathrm{m}$ spacer. A $10 \mathrm{kDa}$ cut-off regenerated cellulose membrane was used as the accumulation wall. The channel pressure has been kept within 5-10 bar by placing a back pressure tubing (approx. $15 \mathrm{~cm}$ long, $750 \mu \mathrm{m}$ ID) between the pumps and the channel. Prior to separation, the membrane was conditioned by soaking it in a water bath filled with pure deionized water overnight. To prevent tube deformation and excessive backpressure on the AF4 system, the original tubes and connectors linking the AF4 to the ICP-MS were replaced with PTFE materials. A flow split system (PTFE T-piece) was inserted post-channel to deliver approximately $10 \%$ of the flow $(0.040 \mathrm{~mL} / \mathrm{min})$ from the AF4 to the ICP-MS to prevent extinguishing of the plasma and carbon deposition on the cones. Both xylene and THF were tested as the carrier liquids. The condensate sample was diluted 1:1 v/v in THF and injected by means of an auto-sampler $(\mathrm{V}=50 \mu \mathrm{L})$. The eluting peaks were detected using a SPD20A UV detector ( $\lambda=325 \mathrm{~nm}$ ) and a PN 3621 (21 angles) multi-angle light scattering (MALS) detector set at 80\% laser power. A constant detector flow rate of $0.5 \mathrm{~mL} / \mathrm{min}$ was maintained throughout the experiment.

The ICP-MS/MS that was coupled to the AF4 system is a 8800 Triple Quadrupole from Agilent Technologies. Elements that were likely to be present in petroleum matrices have been monitored, such as $\mathrm{Al}, \mathrm{Fe}$ and $\mathrm{V}$. The triple quad (ICP-MS/MS) detector allowed the interference-free detection of co-occurring elements present in the condensate sample. Oxygen was introduced in the reaction cell at a rate of $0.3 \mathrm{~L} \cdot \mathrm{min}^{-1}$. $\mathrm{Al}, \mathrm{Co}, \mathrm{Cd}, \mathrm{Hg}$ and $\mathrm{Pb}$ were measured in onmass mode while the other elements were detected in mass-shift mode. The integration time of each targeted $\mathrm{m} / \mathrm{z}$ was 30 ms, and the operating conditions were daily optimized using a tune solution. The optimal separation conditions and ICPMS settings are summarized in Table S3.

\section{RESULTS \& DISCUSSION}

Method development.

AF4 optimization. The optimization step in AF4 appears as the most challenging task because the suitable parameters to be used for the analysis of samples in organic matrices are still poorly known. Performance of the AF4 system for the separation of gas condensates was tested in both xylene and THF. Fig. S1 shows an AF4 fractogram of the separation of 
the Condensate B using xylene and THF as carrier solutions, with the $90^{\circ}$ scattered signal. The first peak appearing around 8 minutes in the figure is a void peak containing unretained material, which corresponds to the time it takes for the carrier solvent to pass through the channel from inlet to outlet. It clearly appears that better separation conditions are met when using THF instead of xylene.

With xylene a small peak at 14 min is observed which is absent in the xylene blank (not shown). With THF, the retention time of the peak observed is the same but with higher intensity. This result seems to show that the retention time, and thus the calculated particle size, is independent from the nature of the carrier solution. However, the higher intensity of the peak observed with THF, demonstrates a better recovery for the particles. Moreover, a smaller additional peak is observed at 12 minutes which potentially indicates a better fractionation and the presence of particles having different sizes. The reasons for these different behaviours are still not clear but may be due to a different stability of the particles in these solvents.

Different cross flow programs were studied. As expected from the FFF theory, higher crossflows resulted in an increase of the retention time (Fig. S2). A starting crossflow rate of $3 \mathrm{~mL} \cdot \mathrm{min}^{-1}$ has been selected in order to increase the resolution between the void peak and the NPs peak.

AF4-ICP-MS coupling. The main limitation of the MALS detector is its inability to provide any elemental information: indeed, it doesn't specifically detect metallic particles as it can also detect polymers. For this reason, the instrument was coupled on-line to an ICP-MS after fractionation. There is an inherent flow limitation when introducing organic solvents (not more than $0.1 \mathrm{~mL} \cdot \mathrm{min}^{-1}$ while the detector flow is $0.5 \mathrm{~mL} \cdot \mathrm{min}^{-1}$ ). Therefore, the output stream has been split by means of a T-piece in order to meet this requirement. The ICP-MS has been initially optimized to work with xylene but the sub-par separation in comparison with THF observed on MALS highlighted the need to optimize it for THF as well. Due to its higher volatility, THF is more likely to form carbon deposits on the ICP cones which is why the flow has been further decreased $\left(0.04 \mathrm{~mL} \cdot \mathrm{min}^{-1}\right)$. This has been done by replacing the tubing connected to the ICP with one of lower diameter. However, the main issue with THF is its tendency to swell PEEK tubing and other plasticlike materials over time ${ }^{26}$. A side effect of the swelling is an increased system pressure which has led to channel overpressure issues in the early stages of method development. Substituting PEEK tubing and ferrules with stainless steel 
counterparts is often recommended in that case, but the presence of $\mathrm{Hg}$ in the sample may be problematic. On the other hand, we have witnessed a marked improvement after switching to PTFE.

Void time, Reproducibility \& Recovery of the AF4 peaks. The void time $\mathrm{t}_{0}$ can be determined using the channel and elution characteristics (detailed calculation shown in Table S3). With the conditions described in the same table, a value of $\mathrm{t}_{0}=0.88$ min was obtained. The Fig. 1 , which shows a MALS overlay of three AF4 fractograms of the Condensate B, presents a small peak around 6 minutes: this indeed corresponds to the focusing time (5 minutes including 1 minute of transition time), plus $\mathrm{t}_{0}$. This peak was therefore identified as the void peak. In order to better assess the performance of the separation, the retention level $\mathrm{R}_{\mathrm{L}}=\mathrm{t}_{\mathrm{r}} / \mathrm{t}_{0}$ is used, where $\mathrm{t}_{\mathrm{r}}$ is the retention time of the analyte (minus the focusing time) $)^{27}$. Therefore, the times will be presented as retention times in this work $\left(t_{r}=t^{0} R_{L}\right)$.

The integrity of the membrane is a key concern in field-flow fractionation analysis. This is even truer when using organic solvents like xylene or THF, since the membrane materials currently used in FFF are not designed for organic applications. Even with aqueous matrices, it is recommended to change the membrane after approximately 30 runs $^{28}$.

As illustrated in Fig. 1 the recovery of the particles peak is gradually increasing as samples are subsequently injected, while the recovery of the void peak is unchanged. This may be an indication that some particles are adsorbed onto the membrane, due to the relatively high crossflow and/or electrostatic interactions. Therefore, the first few sample injections allow to condition the membrane and therefore to increase the subsequent recoveries. The recovery of an injection with the optimized method was assessed by comparing the total peak area given by MALS against the same sample injected without any crossflow (Fig. S3). An average recovery of 99.9\% was observed (triplicates). The recoveries were also determined using the FFF-ICP-MS signal of each monitored element, here again comparing a run with the optimized method against a direct injection (data not shown). It appears that most elements have recoveries around $75-80 \%$. Mercury is a notable exception with a recovery of $22 \%$ : this could indicate that Hg NPs have more affinity with the membrane material, or are too small to be retained.

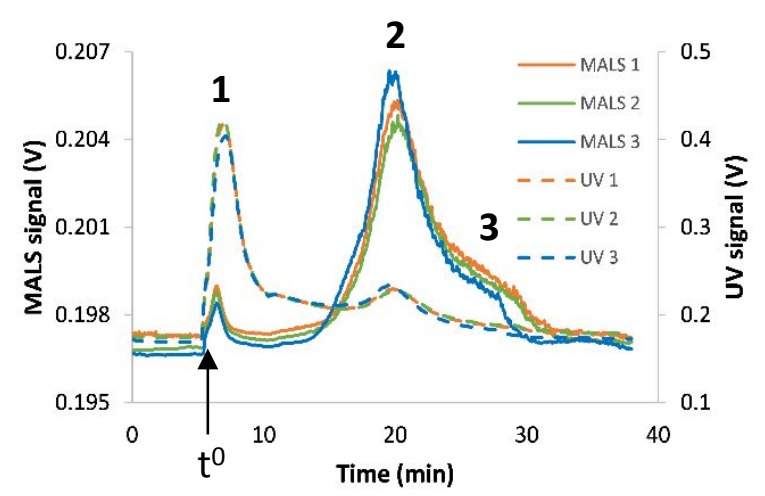


Figure 1. Overlay of AF4 fractograms of the Condensate B in THF with LS- $90^{\circ}$ and UV detection, successively injected in triplicates.

The overlay of MALS and UV fractograms shows that a UV signal is present for both the void peak (peak 1 in Fig. 1) and the main peak (peak 2). The latter $\left(t_{r}=15 \min , R_{L}=17\right)$ also presents a broad shoulder starting from $t_{r}=20$ min (peak 3). However, the UV signal corresponding to peak 2 is much less intense than the void peak, compared with the MALS signal. A strong UV signal often reflects the presence of aromatic and phenolic organic functional groups ${ }^{29}$, which may indicate that more of these compounds are present in the void peak, rather than in peak 2. It is also worth noting that peak 3 shows almost no UV response, which highlights the different compositions of the particles present in the peaks 2 and 3.

\section{Particle size and size distribution.}

STEM. The STEM images were taken at different magnification factors ranging from 20,000 up to 1,000,000 (Fig. 2). It appears that the nanoparticles present in the condensates come in a variety of shapes and are rarely round. In order to gather enough particles to plot the size distribution, 13 images were taken at a magnification factor of 20,000: the size distribution plot obtained with the ImageJ software is shown in Fig. 3. This plot highlights the presence of several populations of nanoparticles, with a major population in number having a diameter of $15-55 \mathrm{~nm}$. There is also a significant proportion of particles between 100 and $200 \mathrm{~nm}$, as well as colloids above $400 \mathrm{~nm}$ size (Fig. 3).
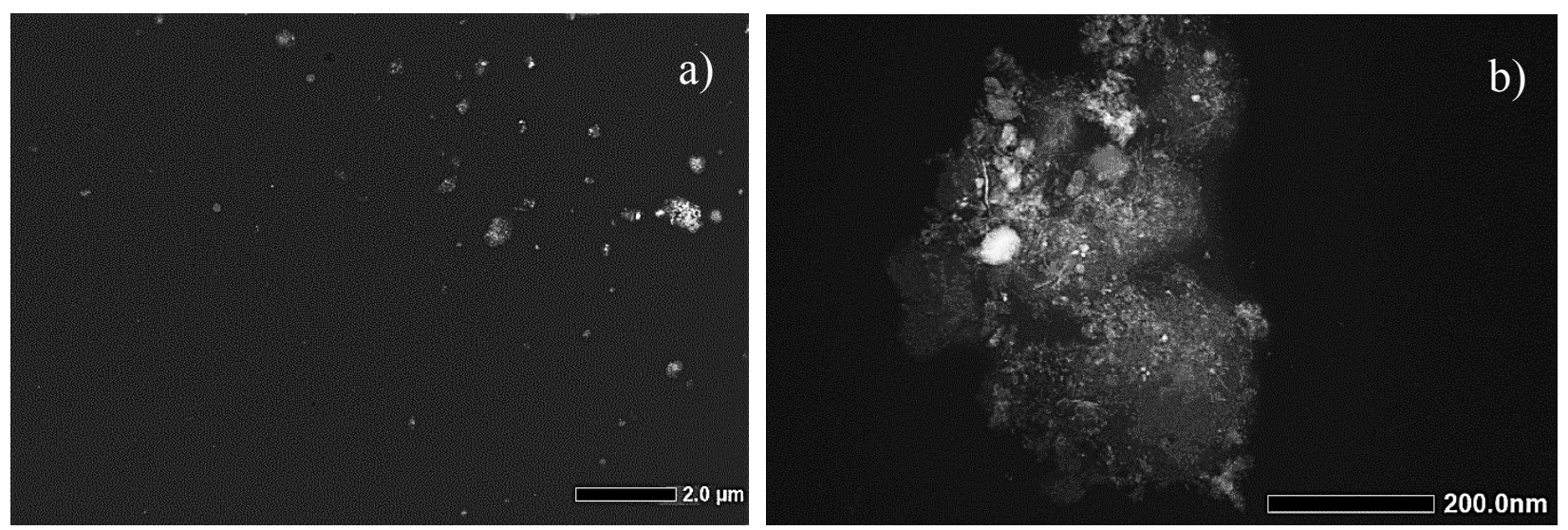

Figure 2. STEM images obtained for the Condensate B. a) x20,000 magnification; b) x250,000 magnification. 


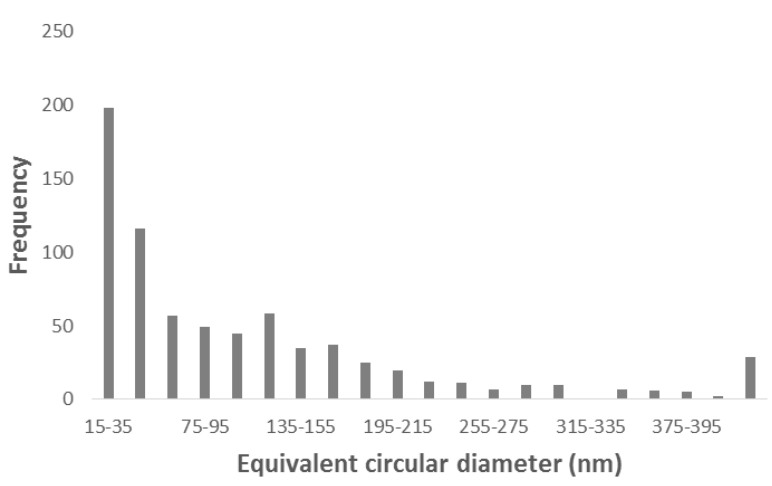

Figure 3. Particle size distribution histograms obtained from the sum of 13 STEM images taken at a x20,000 magnification factor.

AF4-ICP-MS/MS. In order to ensure a good signal to noise ratio, the MALS detector was first normalized with a polystyrene latex standard with a nominal particle diameter of $23 \mathrm{~nm}$. It was not possible to use the $100 \mathrm{~nm}$ Au NPs for this purpose because of the potential enhancement of the light scattering signal due to the surface plasmon resonance effect $^{30}$. The AF4 software was then used to yield the NPs radius of gyration ( $\mathrm{r}_{\mathrm{g}}$ ), using a "random coil" fitting model for the MALS data. This value can then be used to calculate the geometrical radius $\left(\mathrm{r}_{\text {geo }}\right)$ if the particle shape is known: $r_{\text {geo }}=\mathrm{rg} / \alpha$, with $\alpha$ being the shape factor. The standard value of $\alpha=0.775$ has been used for the calculations. This number is a good approximation for spherical particles, which allows to calculate an equivalent circular diameter, but tends to be higher for non-spherical or elongated structures ${ }^{31,32}$. The plot of the calculated $r_{g}$ against elution time for the Condensate B is shown in Fig. 4. It can be seen that the particle size steadily increases from 15-24 minutes $\left(t_{r}=10-19\right.$ min $)$, reaching $\mathrm{r}_{\mathrm{g}}=500 \mathrm{~nm}$ which corresponds to an equivalent circular diameter of $1300 \mathrm{~nm}$. The STEM images also highlighted the presence of different NPs size groups, as well as a significant proportion of NPs above $400 \mathrm{~nm}$. In the light of the $r_{g}$ values calculated using MALS, the AF4 peak seems to contain several size groups as well .The fact that the $\mathrm{r}_{\mathrm{g}}$ decreases around 25 minutes and increases again a few minutes later is surprising and might be an indication that some of the bigger NPs or aggregates break down, possibly due to the pressure inside the channel.

An attempt to integrate the region between 6 and $15 \mathrm{~min}$. ( $\mathrm{t}_{\mathrm{r}}=0$ to $7 \mathrm{~min}$.) has been made. However, none of the available fitting models were suitable for this region, possibly due to the presence of the complex gas condensate matrix in the void peak. Therefore, the $\mathrm{r}_{\mathrm{g}}$ was not calculated. 


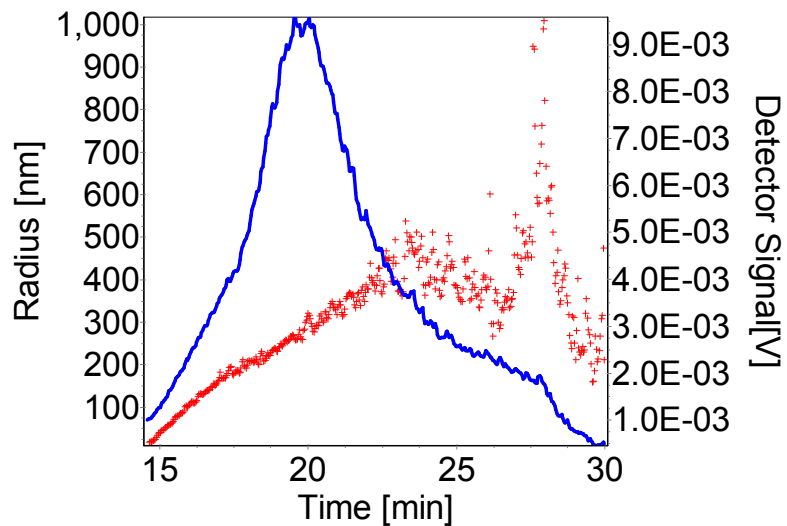

Figure 4. Fractogram of the Condensate B showing the nanoparticles peak between 12 and 20 minutes. The solid line represents the LS-90 ${ }^{\circ}$ signal while the markers indicate the measured radius of gyration $\left(\mathrm{r}_{\mathrm{g}}\right)$ at each time slice.

spICP-MS. In order to confirm the range of particle sizes obtained with AF4, the condensate was analysed offline with spICP-MS, and the data were processed with the MassHunter software. Fig. 5a presents a typical measurement of a condensate sample diluted around 1:1300 in THF. A prevalent $\mathrm{Hg}$ background is observed due to the presence of dissolved $\mathrm{Hg}$ in the condensate. This dilution factor (which corresponds to approximately $3 \times 10^{8}$ particles/L) has been selected as a compromise between a reduced background level and a sufficient number of particles monitored. The relatively small size of the Hg NPs present in the condensate also negatively affects the particles size detection limit because the signal intensity scales as the cube root of the particle diameter. The measurements were done in triplicates, with dilution factors ranging from 1,250 to 1,800 and showed a good reproducibility regarding the size distribution profile (less than 7\% RSD for the median size and 3\% for the mean size). More detailed spICP-MS results are shown in Table S4. The total Hg concentration (NPs + background) calculated by the MassHunter software ranges from 16.2 to $19.6 \mathrm{mg} \cdot \mathrm{kg}^{-1}$, which corresponds to $70-85 \%$ recovery compared to the Hg concentration found by ICP-MS.

However, it must be noted that the particle size can only be accurately determined by spICP-MS if the nature of the nanoparticle is known: indeed, the particle density has to be entered in the spreadsheet for the particle size calculation. Since the latter was first unknown, the calculations were done using the density of $\mathrm{Hg}\left(13.6 \mathrm{~g} \mathrm{~mL}^{-1}\right)$. STEM-EDX data 
later highlighted the presence of HgS NPs (this is discussed more in detail in the "Nature of the nanoparticles" section), which have a density of $8.1 \mathrm{~g} \mathrm{~mL}^{-1}$. Similarly, the particles were assumed to be spherical and the sizes are therefore reported as equivalent circular diameters. The nebulization efficiency, or aerosol transport efficiency, is a key parameter in order to establish the size distribution for a given element ${ }^{33}$. A simple way to estimate the nebulization efficiency is to weigh at a time the amount of waste generated, and to compare it to the amount of liquid left in the initial vial ${ }^{34}$. However, this method has been found to lead to a systematic overestimation of the transport efficiency, due to a significant volume of liquid often being trapped in the spray chamber ${ }^{35,36}$. Therefore, the nebulization efficiency is often determined using a nanoparticle standard of known size and concentration. A typical nebulization efficiency in the case of nanoparticles in an aqueous suspension and a standard type of nebulizer is $2-3 \%{ }^{37}$. However, the microconcentric nebulizers usually have a much higher efficiency, which has been reported to be between 15 and $90 \%$ depending on the nebulizer's internal diameter and the flow rate ${ }^{38-40}$. A nebulization efficiency value of $45 \%$ has been found using the operating conditions in Table S2, and the "particle size" ("RM size") method for calculation (a typical signal obtained for the $100 \mathrm{~nm}$ gold standard is shown in Fig.S4). The Hg NPs have therefore been found to have a median size of 167 $\pm 10 \mathrm{~nm}(192 \pm 11 \mathrm{~nm}$ as $\mathrm{HgS})$, based on three independent measurements (Fig. 5b). The relatively high value found for the median size may be a sign that the smallest HgS NPs cannot be detected by spICP-MS due to the prevalent Hg background.

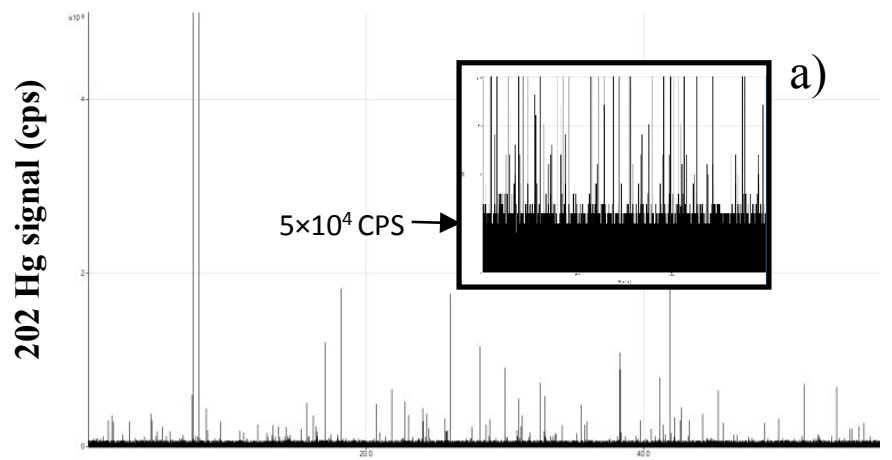

Time (s)

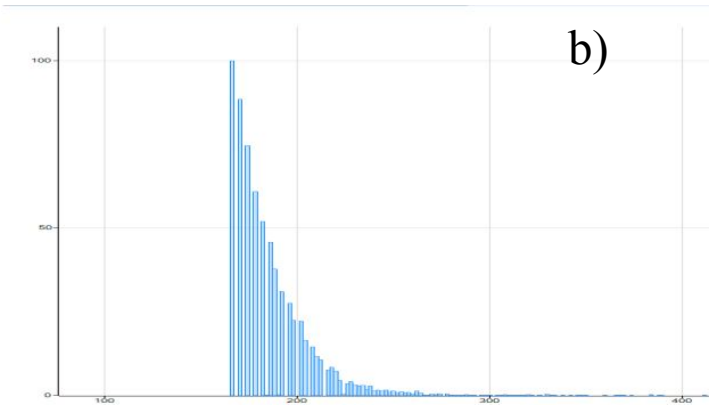

Figure 5. (a) Hg signal for the Condensate B diluted 1:1300 in THF, detected with spICP-MS using a 0.1 ms integration time (inset shows a zoom on the background signal); (b) Particle size distribution obtained using the MassHunter software for the same measurement. 


\section{Nature of the nanoparticles.}

As pointed out previously, the MALS detector is not particle-specific and is unable to give any elemental information. Coupling the AF4 with ICP-MS/MS allowed to overcome this limitation and gave more confidence about the presence of metallic NPs in the condensate sample. As can be seen in Fig. 6, a significant proportion of the metallic content elutes in the void peak. This cannot be seen on MALS, where the void peak is proportionally smaller compared to the NPs peak. The void peak represents less than $5 \%$ of the total fractogram MALS area (around $94 \%$ for the particle peak). In contrast, the peak area of the void peak in ICP-MS/MS accounts for between 10 and $65 \%$ of the total fractogram area, depending on the monitored element. Looking at $\mathrm{Hg}$, it appears that most of the signal (around 65\%) is present in the void peak and up to $t_{r}=7$ minutes. This may indicate the presence of small Hg NPs which elute at very low elution times (from $t_{0}$ to $t_{r}=7$ min.). This phenomenon highlights the usefulness of ICP-MS/MS detection in addition to MALS and UV.

In order to further investigate the nature of the Hg NPs, energy dispersive X-ray (EDX) spectroscopy was used in combination with STEM to generate elemental maps of the sample. In all images, Hg was co-located with S, which is expected due to the high affinity between these two elements. These $\mathrm{HgS} \mathrm{NPs}$ were found to be mostly small, $<100 \mathrm{~nm}$, although some bigger NPs were also present (Fig. S5). These findings are consistent with the AF4-ICP-MS/MS data.

Interestingly, the proportion of material eluting in the second peak compared to the void peak is higher for $\mathrm{Al}$, $\mathrm{Ti}$ and $\mathrm{V}$ than for the other monitored elements. This could be an indication that Al-, Ti- and V-containing NPs are bigger than other metallic NPs in the condensate, and/or have less affinity with the membrane material. The important Al signal could indicate the presence of aluminosilicates, which are commonly found in petroleum matrices ${ }^{41}$. Furthermore, elements such as Ti, As and Fe may be co-located in aluminosilicates ${ }^{42,43}$. The presence of some particles containing Al, $\mathrm{Si}$ and $\mathrm{O}$ in the Condensate B has been confirmed using STEM-EDX (data not shown).

The limitations of spICP-MS when unknown NPs are present in the sample are again highlighted. Indeed, only one element can be monitored at a time unless a TOF detector is used ${ }^{44}$. If the elements are detected one after the other, different NPs will be analyzed which makes any extrapolation challenging. 

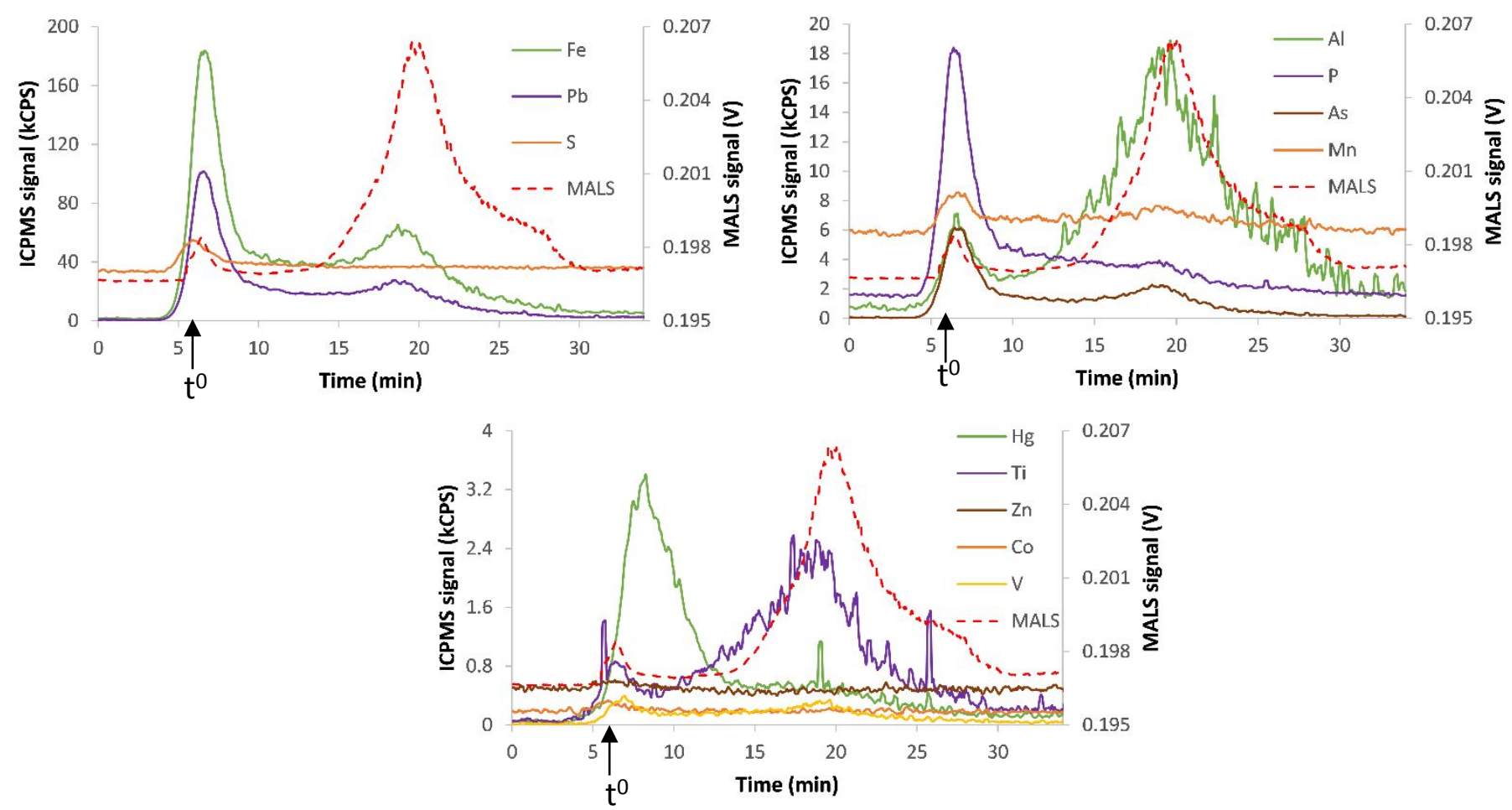

Figure 6. Fractograms of the Condensate B detected on-line with MALS $\left(90^{\circ}\right.$ angle, bottom right) followed by ICP-MS/MS Carrier solution: THF. Fractograms obtained with the new membrane. A retention time shift (approx. 4 minutes) is observed between MALS and ICP-MS/MS signals due to the length and diameter of the connection tubing, and has been corrected for in the figure.

\section{CONCLUSION}

This work demonstrates the applicability of AF4-ICP-MS to obtain qualitative information on the composition of NPs in a gas condensate. The use of THF as a carrier liquid for the AF4 leads to satisfying recoveries compared to o-xylene but can easily damage the standard tubing. This can be overcome (at least temporarily) by using more resistant tubing. The modified cellulose membrane used during this work allows NPs to be separated from the gas condensate matrix, but adsorption of some particles on the membrane is likely to occur, which highlights the need for FlFFF membranes that 
are more suitable for organic matrices. Another drawback of the membranes currently available is that the peaks may not be reproducible upon replacing the worn membrane with a new one ${ }^{45}$.

The STEM images highlighted the presence of irregularly shaped particles and aggregates with a very broad size distribution. The coupling with EDX spectroscopy confirmed the presence of HgS NPs of various sizes in the condensate. Coupled AF4-UV-MALS-ICP-MS/MS confirmed the broad NPs distribution while giving more information about the composition of these natural NPs, as one of the fractions was linked with the presence of several metals as well as aromatic compounds. The spICP-MS confirmed the presence of Hg-containing nanoparticles.

This study highlights the necessity to rely on a whole range of analytical techniques in order to obtain meaningful information about the naturally occurring NPs present in gas condensates. While the MALS and UV detectors are useful to assess the separation efficiency and recovery of the AF4, the lack of elemental information is problematic when element-specific nanoparticles are targeted. The coupling of the AF4 with ICP-MS allowed to monitor not only Hg, but also other metals in the nanoparticulate fraction.

\section{SUPPORTING INFORMATION}

The supporting information includes the optimised AF4-ICPMS and spICP-MS parameters, additional AF4 and spICP-MS figures, as well as the STEM-EDX data.

\section{ACKNOWLEDGEMENTS}

Dr. Andrea Raab (University of Aberdeen, U.K.) is thanked for her advice on ICP-MS, which has helped to achieve this work. D.R. thanks Johnson Matthey, U.K. for the provided studentship; special thanks go to Colin Baptist, Lucy Barrass, Matt Lunn, Stefano Martinuzzi, Katie Smart and Bradley Waldron for the fruitful discussions and support; as well as Emily Brooke for her help with the STEM-EDX analyses. K.N. acknowledges the support provided by the University of Aberdeen through the Elphinstone scholarship. Authors also gratefully acknowledge Postnova Analytics UK for the loan of the AF4 system together with training, support and advice on the technique. Special thanks go to Dr. Bassem Sabagh for his help. 
D.R. and K.N. contributed equally to this work.

\section{CONFLICTS OF INTEREST}

There are none to declare.

\section{REFERENCES}

1. Pyziur, M. Condensate - An EPRINC Primer. (2015). Available at: https://eprinc.org/wpcontent/uploads/2015/02/EPRINC-Condensate-Primer.pdf (Accessed: 5th July 2017).

2. Sarrazin, P., Cameron, C. J., Barthel, Y. \& Morrison, M. E. Processes Prevent Detrimental Effects from As and Hg in Feedstocks. Oil Gas J. 91, 86-90 (1993).

3. Filby, R. H. Origin and nature of trace element species in crude oils, bitumens and kerogens: implications for correlation and other geochemical studies. Geol. Soc. London, Spec. Publ. 78, 203-219 (1994).

4. Wilhelm, S. M. Risk analysis for operation of aluminum heat exchangers contaminated by mercury. Process Saf. Prog. 28, 259-266 (2009).

5. Dunleavy, J. K. Mercury as a catalyst poison. Platin. Met. Rev. 50, 156 (2006).

6. Johnson Matthey. Functional Speciation of Mercury in Condensate (unpublished internal document). (2016).

7. Sainal, M. R., Carigali, P., Bhd, S., Shafawi, A. \& Services, S. Mercury Removal System for Upstream Application : Experience in Treating Mercury From Raw Condensate SPE-106610. E\&P Environ. Saf. Conf. 1-10 (2007). doi:10.2118/106610-MS

8. Gajdosechova, Z., Boskamp, M. S., Lopez-Linares, F., Feldmann, J. \& Krupp, E. M. Hg Speciation in Petroleum Hydrocarbons with Emphasis on the Reactivity of Hg Particles. Energy \& Fuels 30, 130-137 (2016).

9. Ezzeldin, M. F. et al. Mercury speciation and distribution in an Egyptian natural gas processing plant (in press). Energy \& Fuels (2016).

10. Avellan, A. et al. Speciation of Mercury in Selected Areas of the Petroleum Value Chain. Environ. Sci. Technol. 52, 1655-1664 (2018).

11. Montaño, M. D., Olesik, J. W., Barber, A. G., Challis, K. \& Ranville, J. F. Single Particle ICP-MS: Advances toward routine analysis of nanomaterials. Anal. Bioanal. Chem. 408, 5053-5074 (2016).

12. Hassellöv, M., Readman, J. W., Ranville, J. F. \& Tiede, K. Nanoparticle analysis and characterization methodologies in environmental risk assessment of engineered nanoparticles. Ecotoxicology 17, 344-361 (2008).

13. Laborda, F. et al. Detection, characterization and quantification of inorganic engineered nanomaterials: A review of techniques and methodological approaches for the analysis of complex samples. Anal. Chim. Acta 904, 10-32 (2016).

14. Laborda, F., Bolea, E. \& Jimenez-Lamana, J. Single Particle Inductively Coupled Plasma Mass Spectrometry: A Powerful Tool for Nanoanalysis. Anal. Chem. 86, 2270-2278 (2014).

15. Wells, M. L. The colloidal size spectrum of CDOM in the coastal region of the Mississippi Plume using flow fieldflow fractionation. Mar. Chem. 89, 89-102 (2004).

16. Stolpe, B., Guo, L., Shiller, A. M. \& Hassellöv, M. Size and composition of colloidal organic matter and trace elements in the Mississippi River, Pearl River and the northern Gulf of Mexico, as characterized by flow field-flow fractionation. Mar. Chem. 118, 119-128 (2010).

17. Andersson, M., Wittgren, B. \& Wahlund, K.-G. Ultrahigh Molar Mass Component Detected in Ethylhydroxyethyl Cellulose by Asymmetrical Flow Field-Flow Fractionation Coupled to Multiangle Light Scattering. Anal. Chem. 73, 4852-4861 (2001).

18. von der Kammer, F., Baborowski, M. \& Friese, K. Field-flow fractionation coupled to multi-angle laser light scattering detectors: Applicability and analytical benefits for the analysis of environmental colloids. Anal. Chim. Acta 552, 166174 (2005). 
19. Bang, D. Y., Shin, D. Y., Lee, S. \& Moon, M. H. Characterization of functionalized styrene-butadiene rubber by flow field-flow fractionation/light scattering in organic solvent. J. Chromatogr. A 1147, 200-205 (2007).

20. Hassellöv, M., Lyvé, B., Haraldsson, C. \& Sirinawin, W. Determination of Continuous Size and Trace Element Distribution of Colloidal Material in Natural Water by On-Line Coupling of Flow Field-Flow Fractionation with ICPMS. Anal. Chem. 71, 3497-3502 (1999).

21. De La Calle, I., Pérez-Rodríguez, P., Soto-Gómez, D. \& López-Periago, J. E. Detection and characterization of Cubearing particles in throughfall samples from vine leaves by DLS, AF4-MALLS (-ICP-MS) and SP-ICP-MS. Microchem. J. 133, 293-301 (2017).

22. Prestel, H., Schott, L., Niessner, R. \& Panne, U. Characterization of sewage plant hydrocolloids using asymmetrical flow field-flow fractionation and ICP-mass spectrometry. Water Res. 39, 3541-3552 (2005).

23. Mehn, D. FFF-MALS method development and measurements of size and molecular weight. (EUNCL, 2016).

24. Kirkland, J. J., Dilks, C. H. \& Dupont, E. I. Flow Field-Flow Fractionation of Polymers in Organic Solvents. Anal. Chem 64, (1992).

25. Poirier, L. et al. Application of ICP-MS and ICP-OES on the Determination of Nickel, Vanadium, Iron, and Calcium in Petroleum Crude Oils via Direct Dilution. (2016). doi:10.1021/acs.energyfuels.5b02997

26. Meyer, V. R. Practical High-Performance Liquid Chromatography Fourth Edition. (John Wiley, 2004).

27. Wahlund, K.-G. Flow field-flow fractionation: Critical overview. J. Chromatogr. A 1287, 97-112 (2013).

28. Moquin, A., Winnik, F. M. \& Maysinger, D. in Methods in Molecular Biology (ed. Weissig, V.) 991, 325-340 (Springer, 2013).

29. Weishaar, J. L. et al. Evaluation of Specific Ultraviolet Absorbance as an Indicator of the Chemical Composition and Reactivity of Dissolved Organic Carbon. Environ. Sci. Technol. 37, 4702-4708 (2003).

30. Fan, X., Zheng, W. \& Singh, D. J. Light scattering and surface plasmons on small spherical particles. Light Sci. Appl. 3, (2014).

31. Brewer, A. K. \& Striegel, A. M. Particle size characterization by quadruple-detector hydrodynamic chromatography. Anal. Bioanal. Chem. 393, 295-302 (2009).

32. Qi, W. H., Wang, M. P. \& Liu, Q. H. Shape factor of nonspherical nanoparticles. J. Mater. Sci. 40, 2737-2739 (2005).

33. Degueldre, C. \& Favarger, P.-Y. Colloid analysis by single particle inductively coupled plasma- mass spectroscopy: a feasibility study. Colloids Surfaces A 217, 137-142 (2003).

34. Kato, K. Indirect measuring method of aerosol transport efficiency in inductively coupled plasma spectrometry. Fresenius Z. Anal. Chem. 329, 861-863 (1988).

35. Smith, D. D. \& Browner, R. F. Measurement of Aerosol Transport Efficiency in Atomic Spectrometry. Anal. Chem. 54, 533-537 (1982).

36. Pace, H. E. et al. Determining transport efficiency for the purpose of counting and sizing nanoparticles via single particle inductively coupled plasma-mass spectrometry. Anal. Chem. 83, 9361-9369 (2011).

37. RIKILT - Institute of food safety. Nanoparticle Suspensions - Counting and Sizing Nanoparticles Via Single Particle Inductively Coupled Plasma Mass Spectrometry. (2013).

38. Hettipathirana, T. D. \& Davey, D. E. Evaluation of a microconcentric nebuliser- cyclonic spray chamber for flow injection simultaneous multielement inductively coupled plasma optical emission spectrometry. J. Anal. At. Spectrom. 13, 483-488 (1998).

39. Cairns, W. R. L. et al. Performance characteristics of a low volume spray chamber with a micro-flow nebulizer for ICP-MS. J. Anal. At. Spectrom. 19, 286-291 (2004).

40. Kanaki, K. \& Pergantis, S. A. Using nanoparticles to determine the transport efficiency of microflow and nanoflow nebulizers in inductively coupled plasma-mass spectrometry. J. Anal. At. Spectrom. 31, 1041-1046 (2016).

41. Johns, W. D. Clay Mineral Catalysis and Petroleum Generation. Ann. Rev. Earth Planet. Sci 7, 183-98 (1979).

42. García-Sánchez, A., Alastuey, A. \& Querol, X. Heavy metal adsorption by different minerals: application to the remediation of polluted soils. The Science of the Total Environment 242, (1999).

43. Guignard, M., Cormier, L., Montouillout, V., Menguy, N. \& Massiot, D. Structural fluctuations and role of Ti as nucleating agent in an aluminosilicate glass. J. Non. Cryst. Solids 356, 1368-1373 (2010).

44. Balcerzak, M. An overview of analytical applications of time of flight-mass spectrometric (TOF-MS) analyzers and 
an inductively coupled plasma-TOF-MS technique. Anal. Sci. 19, 979-989 (2003).

45. Meisterjahn, B., Wagner, S., von der Kammer, F., Hennecke, D. \& Hofmann, T. Silver and gold nanoparticle separation using asymmetrical flow-field flow fractionation: Influence of run conditions and of particle and membrane charges. J. Chromatogr. A 1440, 150-159 (2016).

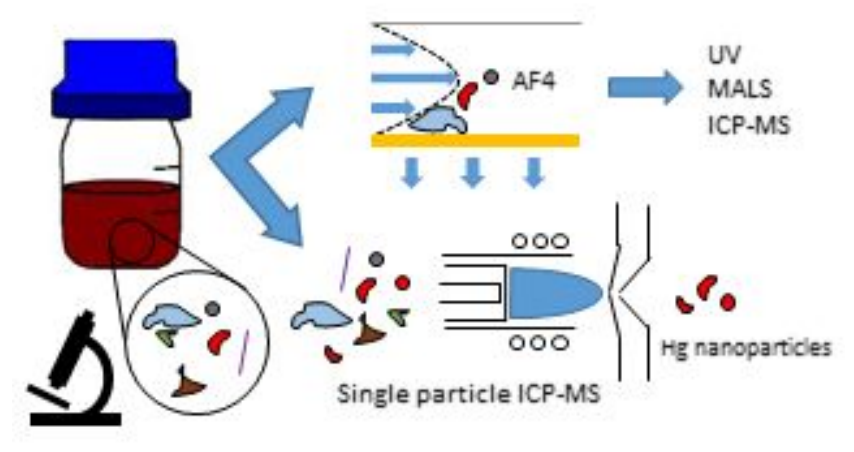

Table of Contents artwork 